\title{
ESTUDO COMPARATIVO ACERCA DA ESCOLHA DO CURSO DE PEDAGOGIA EM INSTITUIÇÕES PARTICULARES DE SÃO PAULO
}

\author{
Nonato Assis de Miranda ${ }^{1}$ \\ Sandra Farto Botelho Trufem ${ }^{2}$ \\ Jomar Barros Filho ${ }^{3}$
}

\begin{abstract}
RESUMO: este trabalho analisa comparativamente os motivos da escolha de curso e as concepções sobre a carreira percebidas por alunos do curso de Pedagogia. Trata-se de pesquisa de natureza quantitativa realizada com 296 estudantes do período noturno em instituições de natureza privada na Grande São Paulo. Para atender ao objetivo proposto, realizou-se o teste de Kruskal-Wallis no sentido de comparar as concepções dos alunos nos diferentes campi. Os resultados apontam que estudantes de diferentes regiões e contextos sociais não apresentam opiniões muito divergentes a respeito da escolha do curso e do campo de atuação profissional do Pedagogo.
\end{abstract}

Palavras-chave: curso de Pedagogia; escolha profissional; ensino superior noturno; teste de Kruskal-Wallis.

\section{INTRODUÇÃO}

A escolha profissional é assunto que, apesar de ser debatido há algum tempo no Brasil, como é o caso dos trabalhos realizados por Lehman (1999), Macedo (1989), Weil (1991), Silva (1996), Whitaker (1997), Bock (2001) e muitos outros, não deixa de ser importante, pois dependendo do contexto e da abordagem escolhida para se estudar poderá trazer novas contribuições à sociedade.

A respeito do assunto, Enge (2004) afirma que o que freqüentemente é denominado escolha profissional é, na verdade, um processo complexo e contínuo, através do qual, os indivíduos buscam, constantemente, compatibilizar suas próprias aspirações e desejos com as oportunidades do mercado de trabalho. A autora acrescenta que, em geral, o conceito de profissão é empregado no senso comum. Com isso, percebeu-se que o termo utilizado pelos especialistas da área é profissão e as suas derivações. Neste caso, de modo similar trata-se com exclusividade da escolha de uma profissão universitária.

Em face ao exposto, esse trabalho objetiva analisar as concepções de alunos de diferentes contextos sociais acerca da escolha do curso de Pedagogia em instituições de natureza privada da Grande São Paulo.

\section{PERCURSO METODOLÓGICO}

\footnotetext{
${ }^{1}$ Universidade Estadual de Campinas-Unicamp/UNIP.

${ }^{2}$ Universidade São Marcos

${ }^{3}$ Universidade São Marcos
} 
Essa investigação caracteriza-se por ser pesquisa quantitativa. A vantagem desse método é o de se levantar informações com confiabilidade estatística. De acordo Demo (2000), trata-se do emprego da quantificação da coleta de dados e o tratamento destes por meio de técnicas estatísticas (simples ou complexas).

Os dados foram obtidos por meio de levantamento amostral (survey), com escala de atitudes de Likert realizado em amostra composta por 296 alunos do curso de Pedagogia em três campi de uma Universidade e de um Centro Universitário, ambos do setor privado.

Para Mattar (1997), uma escala Likert, proposta por Rensis Likert em 1932, é uma escala na qual os respondentes são solicitados não só a concordarem ou discordarem das afirmações, mas também a informarem qual o seu grau de concordância ou de discordância.

$\mathrm{Na}$ tentativa de comparar as concepções de alunos de diferentes contextos sociais empregou-se o teste de Kruskal-Wallis. De acordo com Siegel (1981), esse teste é uma prova extremamente útil para decidir se $\mathrm{k}$ amostras independentes provêm de populações diferentes. $\mathrm{O}$ autor afirma que os valores amostrais quase que invariavelmente diferem entre si, e o problema é decidir se essas diferenças entre as amostras significam diferenças efetivas entre as populações, ou se representam apenas variações casuais, que podem ser esperadas entre amostras aleatórias de uma mesma população.

Para Pestana e Gageiro (2000), o teste de Kruskal-Wallis baseia-se na soma das ordens dos grupos combinados por ordem crescente. A menor observação tem o valor 1 , a seguinte $o$ valor 2 e assim sucessivamente. Caso existam observações com o mesmo valor (empates) atribui-lhes o valor médio, dado pela posição seqüencial das observações que lhe corresponderiam caso não houvesse empate. Os autores apontam que, caso haja apenas 3 grupos com dimensão menor ou igual a 5, utilizam-se as tabelas de Krukal-Wallis para se calcular o valor do teste. Em todas as outras situações faz-se a aproximação à distribuição do Qui-Quadrado com (k-1) graus de liberdade.

\section{APRESENTAÇÃO DOS RESULTADOS}

Para que se possa proceder a este tipo de análise, ou seja, a aplicação do teste de Krusal-Wallis, deve-se dividir os sujeitos em categorias prévias. Para isso, optou-se pela separação por campi levando-se em consideração a ordem da coleta dos dados, ou seja, campus 1 Santo Amaro, campus 2 Alphaville, campus 3 Tatuapé e campus 4 Vila Mariana.

De acordo com Pestana e Gageiro (2000), para que se possa realizar o teste de Kruskal-Wallis, deve-se verificar hipótese de não normalidade dos dados. Desta forma, realizou-se o teste de Kolmogorv-Smirnov (Tabela 1).

Tabela 1: Teste de Normalidade dos dados feito a partir do teste de Kolmogorov-Smirnov.

Kolmogorov-Smirnov

Variável

Estatística

Significância 


\begin{tabular}{|c|c|c|}
\hline \multicolumn{3}{|c|}{ Kolmogorov-Smirnov } \\
\hline 1 & 7,057 &, $000^{4}$ \\
\hline 2 & 6,906 &, 000 \\
\hline 3 & 5,879 &, 000 \\
\hline 4 & 5,667 & ,000 \\
\hline 5 & 2,984 &, 000 \\
\hline 6 & 2,890 &, 000 \\
\hline 7 & 2,964 &, 000 \\
\hline 8 & 6,254 &, 000 \\
\hline 9 & 4,817 & ,000 \\
\hline 10 & 5,082 & ,000 \\
\hline 11 & 4,133 & ,000 \\
\hline 12 & 4,211 & ,000 \\
\hline 13 & 3,659 & ,000 \\
\hline 14 & 3,028 & ,000 \\
\hline 15 & 5,259 & ,000 \\
\hline 16 & 4,468 & ,000 \\
\hline 17 & 2,849 & ,000 \\
\hline 18 & 3,147 & ,000 \\
\hline 19 & 4,103 & ,000 \\
\hline 20 & 3,563 & ,000 \\
\hline 21 & 5,210 &, 000 \\
\hline 22 & 5,916 & ,000 \\
\hline 23 & 5,244 & ,000 \\
\hline 24 & 4,704 &, 000 \\
\hline 25 & 3,146 &, 000 \\
\hline 26 & 3,670 &, 000 \\
\hline 27 & 4,386 &, 000 \\
\hline 28 & 4,035 & ,000 \\
\hline 29 & 3,341 & ,000 \\
\hline 30 & 6,633 & 000 \\
\hline 31 & 4,176 & ,000 \\
\hline 32 & 3,194 & ,000 \\
\hline 33 & 3,709 &, 000 \\
\hline 34 & 4,048 & ,000 \\
\hline 35 & 5,472 & ,000 \\
\hline 36 & 4,888 & ,000 \\
\hline 37 & 2,773 &, 000 \\
\hline 38 & 3,577 & 000 \\
\hline 39 & 3,469 & ,000 \\
\hline 40 & 5,060 & 000 \\
\hline
\end{tabular}

Fonte: Método de Extração: Análise Fatorial de Componentes Principais.

Como a significância é menor que 0,0001, pode-se concluir que as distribuições de todas as variáveis não são normais ao nível de significância de 0,05 conforme advogam Pestana e Gageiro (2000). Portanto, os dados são não-paramétricos e o teste de KruskalWallis pode ser aplicado.

4 Para interpretar esse resultado, Maria Helena Pestana e João Nunes Cageiro (2000), entendem que significâncias inferiores a 0,0001 como estas, sugerem que as distribuições de todas as variáveis não são normais ao nível de significância de 0,05 . 
A seguir, os sujeitos foram divididos por região de pesquisa para verificar se sujeitos de três bairros e um município da Grande São Paulo possuem opiniões diferentes em relação às assertivas do instrumento de pesquisa. Para testar esta hipótese realizou-se o teste de Kruskall-Walllis. Para tanto, os sujeitos foram divididos em quatro grupos: Grupo 1 - Santo Amaro, Grupo 2 - Alphaville, Grupo 3 - Tatuapé, Grupo 4 - Vila Mariana.

O teste de Kruskal-Wallis para esta variável pode ser observado na Tabela 2.

Tabela 2: Teste de Kruskal-Wallis para a variável campus de estudo.

\begin{tabular}{|c|c|c|c|}
\hline Variável & Chi-Quadrado & DF & Significância \\
\hline 1 & 4,855 & 3 &, 183 \\
\hline 2 & 5,052 & 3 &, 168 \\
\hline 3 & 6,180 & 3 & 103 \\
\hline 4 & 6,797 & 3 & 079 \\
\hline 5 & 1,368 & 3 &, 713 \\
\hline 6 &, 652 & 3 & 885 \\
\hline 7 & 5,088 & 3 & 165 \\
\hline 8 &, 825 & 3 & 843 \\
\hline 9 & 651 & 3 & 885 \\
\hline 10 & 1,023 & 3 & ,796 \\
\hline 11 & 9,211 & 3 &, 027 \\
\hline 12 & 2,550 & 3 & ,466 \\
\hline 13 & 3,441 & 3 & 328 \\
\hline 14 & 9,760 & 3 & ,021 \\
\hline 15 & ,683 & 3 & 877 \\
\hline 16 & 4,405 & 3 & 221 \\
\hline 17 & 14,315 & 3 &, 003 \\
\hline 18 & 4,710 & 3 & 194 \\
\hline 19 & 1,489 & 3 & 685 \\
\hline 20 & 3,533 & 3 &, 316 \\
\hline 21 & 3,018 & 3 & 389 \\
\hline 22 & 4,641 & 3 & 200 \\
\hline 23 & ,497 & 3 & ,920 \\
\hline 24 & 5,315 & 3 & 150 \\
\hline 25 & 1,499 & 3 & 682 \\
\hline 26 & 13,529 & 3 & ,004 \\
\hline 27 & 7,306 & 3 & ,063 \\
\hline 28 & 5,839 & 3 & 120 \\
\hline 29 & 15,893 & 3 & ,001 \\
\hline 30 & 1,646 & 3 & 649 \\
\hline 31 & 11,285 & 3 & ,010 \\
\hline 32 & 4,279 & 3 & 233 \\
\hline 33 & 13,785 & 3 & ,003 \\
\hline 34 & 7,401 & 3 & ,060 \\
\hline 35 & ,287 & 3 & 962 \\
\hline 36 & 3,831 & 3 & 280 \\
\hline 37 & 14,025 & 3 &, 003 \\
\hline 38 & 8,296 & 3 &, 040 \\
\hline
\end{tabular}




\begin{tabular}{cccc}
\hline \multirow{2}{*}{ Variável } & Chi-Quadrado & DF & Significância \\
\hline 39 & 3,858 & 3 &, 277 \\
40 & 5,869 & 3 &, 118 \\
\hline
\end{tabular}

Fonte: Método de Extração: Análise Fatorial de Componentes Principais.

Pode-se observar na Tabela 2, que as variáveis (anexo 1) 11, 14, 17, 26, 29, 31, 33, 37 e 38, destacadas na tabela, possuem níveis de significância baixos (menores que 0,05). Para Pestana e Gageiro (2000), isso significa que pelo menos um dos grupos difere dos restantes em relação a estas variáveis.

Segundo Siegel (1981), para as outras variáveis, as diferenças obtidas nas respostas dos sujeitos de cada grupo que não são estatisticamente significantes, representam apenas variações casuais. As diferenças entre os grupos para as variáveis mencionadas acima podem ser percebidas pela tabela 3 , sendo que todos os dados são apresentados em percentagens, e D $=$ discordo ou discordo plenamente $\mathrm{I}=$ indiferente $\mathrm{e} \mathrm{C}=$ concordo ou concordo plenamente.

Tabela 3: Diferenças entre os grupos da variável área de pesquisa.

\begin{tabular}{|c|c|c|c|c|c|c|c|c|c|c|c|c|}
\hline \multirow[t]{2}{*}{ Variáveis } & \multicolumn{3}{|c|}{$\begin{array}{l}\text { Santo } \\
\text { Amaro }\end{array}$} & \multicolumn{3}{|c|}{ Alphaville } & \multicolumn{3}{|c|}{ Tatuapé } & \multicolumn{3}{|c|}{$\begin{array}{c}\text { Vila } \\
\text { Mariana }\end{array}$} \\
\hline & $\mathrm{D}$ & I & $\mathrm{C}$ & $\mathrm{D}$ & I & $\mathrm{C}$ & $\mathrm{D}$ & I & $\mathrm{C}$ & $\mathrm{D}$ & I & $\mathrm{C}$ \\
\hline 11 & 9,9 & 22,0 & 68,1 & 4,7 & 10,9 & 84,4 & 3,3 & 13,3 & 83,3 & 7,5 & 4,5 & 88,1 \\
\hline 14 & 34,0 & 25,5 & 40,4 & 21,9 & 31,3 & 46,9 & 30,2 & 23,8 & 46,0 & 16,2 & 22,1 & 61,8 \\
\hline 17 & 38,7 & 29,0 & 32,3 & 24,6 & 41,5 & 33,8 & 38,7 & 27,4 & 33,9 & 17,6 & 27,9 & 54,4 \\
\hline 26 & 64,4 & 18,9 & 16,7 & 53,0 & 25,8 & 21,2 & 61,9 & 23,8 & 14,3 & 79,7 & 13,0 & 7,2 \\
\hline 29 & 33,3 & 13,3 & 53,3 & 17,2 & 14,1 & 68,8 & 43,8 & 21,9 & 34,4 & 34,8 & 13,0 & 52,2 \\
\hline 31 & 10,3 & 18,4 & 71,3 & 9,7 & 12,9 & 77,4 & 34,9 & 9,5 & 55,6 & 21,5 & 13,8 & 64,6 \\
\hline 33 & 24,4 & 14,4 & 61,1 & 11,5 & 13,1 & 75,4 & 41,0 & 18,0 & 41,0 & 23,1 & 20,0 & 56,9 \\
\hline 37 & 21,3 & 18,0 & 60,7 & 22,4 & 43,1 & 34,5 & 41,0 & 27,9 & 31,1 & 26,2 & 27,7 & 46,2 \\
\hline 38 & 42,7 & 9,0 & 48,3 & 31,3 & 14,1 & 54,7 & 56,5 & 14,5 & 29,0 & 45,5 & 12,1 & 42,4 \\
\hline
\end{tabular}

Fonte: Método de Extração: Análise Fatorial de Componentes Principais.

Pela tabela 3, pode-se perceber que a maioria em todos os grupos concorda com as assertivas 11, 14 e 31, embora haja uma certa diferença na percentagem de sujeitos que concordam. Na assertiva 11 (o curso é voltado para a área das humanidades), os sujeitos de Santo Amaro tendem a expressar uma percentagem bem menor de concordâncias que os outros grupos. Já o grupo do Tatuapé possui menor quantidade de concordâncias em relação aos demais grupos na assertiva 31 (é de fácil acesso).

Em relação à assertiva 14 (para poder trabalhar na área de treinamento de pessoal), percebe-se que o campus da Vila Mariana possui uma maior percentagem de sujeitos 
expressando concordância do que os demais grupos. Observou-se também que todos os grupos discordam da assertiva 26 (é um curso fácil), sendo que o grupo de Alphaville expressa um menor número de discordâncias e os sujeitos da Vila Mariana expressam o maior número.

No entanto, as diferenças mais marcantes são encontradas nas assertivas 17, 29, 33, 37 e 38. Os grupos de Santo Amaro e Tatuapé, em sua maioria, discordam da assertiva 17 (para trabalhar em treinamento empresarial) enquanto que os sujeitos da Vila Mariana tendem a concordar com esta assertiva. Por outro lado, para os alunos de Alphaville, esta assertiva tende a ser indiferente. Em relação à variável 29 (quero melhorar meu salário, que diz respeito à evolução funcional), o grupo do Tatuapé tende a discordar da assertiva enquanto que os demais a concordar.

Para a variável 33 (está localizada perto da minha casa), constatou-se que o grupo do Tatuapé expressa a mesma percentagem de concordâncias e discordâncias, enquanto que os outros grupos expressam, em sua maioria, concordância com a assertiva.

Já os grupos Santo Amaro e Vila Mariana tendem a concordar com a variável 37 (é uma universidade e não faculdade), enquanto que os sujeitos do Tatuapé tendem a discordar e os de Alphaville são indiferentes. Em relação à variável 38 (tenho bolsa), constatou-se que os sujeitos de Santo Amaro e Alphaville expressam concordância com esta assertiva e os demais grupos expressam discordância.

\section{CONCLUSÕES}

$\mathrm{Na}$ tentativa de comparar as opiniões de estudantes universitários de Pedagogia de diferentes regiões da Grande São Paulo, os resultados apontaram para o consenso em vários aspectos. Por outro lado, há algumas divergências de pensamento entre os respondentes. Em relação às concordâncias, encontram-se as assertivas que indagavam se o curso de Pedagogia é fácil; se está voltado para as humanidades (os sujeitos do campus de Santo Amaro apontaram tendência de concordância inferior aos demais grupos); se escolheram o curso para trabalhar na área de treinamento pessoal.

Todavia, algumas discrepâncias foram verificadas. Ao serem indagados se haviam escolhido o curso para trabalharem na área de treinamento empresarial, os estudantes dos campi de Santo Amaro e Tatuapé discordaram, ao passo que os de Alphaville foram indiferentes aos assunto (talvez não souberam manifestar a respeito por desconhecimento do assunto) e os da Vila Mariana concordaram.

Quando questionados se haviam escolhido o curso em busca de eventual melhoria de salários, como evolução funcional via acadêmica, por exemplo, os respondentes apresentaram respostas diferenciadas, pois enquanto os representantes de Santo Amaro, Alphaville e Vila Mariana concordaram com a assertiva, 43,8\% dos estudantes do Tatuapé discordaram da indagação. Outro questionamento que não teve unanimidade foi em relação ao fato de terem escolhido a instituição porque haviam recebido bolsa. Os resultados mostram que os estudantes da Universidade, em seus três campi pesquisados, concordaram com essa indagação, ao passo que o grupo da Vila Mariana, ou seja, do Centro Universitário discordaram. 
Neste caso, vale mencionar que os resultados são prudentes, pois a Universidade oferece bolsa com iniciativa própria, além de convênios que mantém com a Associação de Pais do Estado de São Paulo e o Governo do Estado de São Paulo (Escola da Família). Por outro lado, no caso do Centro Universitário pesquisado, a oferta de bolsa se dá por iniciativa da própria IES e somente a partir do segundo ano. Como a pesquisa foi realizada com alunos do primeiro e do segundo semestres, os mesmos não haviam sido beneficiados pela bolsa de estudo.

Portanto, em face do exposto, a hipótese que orientou a realização do teste de kruskalWallis, ou seja, alunos de diferentes regiões têm as mesmas concepções em relação à escolha profissional, se corrobora, pois não há consenso entre os sujeitos investigados nos diferentes campi.

Ao finalizar o presente estudo, coloca-se, mais uma vez, num ponto de partida. Os questionamentos que alimentaram as discussões produziram concepções que estão além da subjetividade da escolha profissional. Percebe-se que não basta procurar explicitar um fenômeno pelo que se acredita e pelo que os dados empíricos possam eventualmente mostrar, pois as incertezas que percorrem o curso de Pedagogia desde a sua criação na década de 1930 parecem assombrar os formandos do mesmo curso em pleno século XXI.

\section{REFERÊNCIAS BIBIBLIOGRÁFICAS}

BOCK, Silvio Duarte. (2001). Orientação Profissional: avaliação de uma proposta de trabalho na avaliação sócio-histórica. Dissertação de mestrado em Psicologia da Educação - Faculdade de Educação da Unicamp.

ENGE, Janine Schultz. (2004). Da universidade ao mundo do trabalho: um estudo sobre o início da profissionalização de egressos do curso de licenciatura da USP (1994-1995). Dissertação de Mestrado em educação - Faculdade de Educação da USP.

LEHMAN, Ivette Piha. (1999). Não sei que profissão escolher. São Paulo: Moderna. MACEDO, Roberto Brás Matos. (1998). Seu diploma, sua prancha: como escolher a profissão e surfar no mercado de trabalho. 7 ed. São Paulo: Saraiva.

MATTAR, F. (1997). Pesquisa de Marketing. São Paulo: Atlas.

PESTANA, M.H.; GAGEIRO, J.N. (2000). Análise de dados para ciências sociais: a complementaridade do SPSS. 2 ed. Lisboa: Edições Silabo.

SILVA, Laura B. de Campos. (1996). A escolha da profissão: uma abordagem psicossocial. São Paulo: Unimarco.

SIEGEL, S. (1981). Estatística não - paramétrica. São Paulo: Editora McGraw-Hill do Brasil Ltda.

WEIL, Pierre. (1991). Sua vida, seu futuro - escolha entre 600 profissões. 13 ed. Petrópolis: Vozes.

WHITAKER, Dulce. (1997). Escolha da carreira e globalização. 11 ed. São Paulo: Moderna.

\section{Anexo 1 - Questionário elaborado para coleta de dados.}

Este questionário foi elaborado com a finalidade de levantar as concepções de alunos sobre o curso. Ao respondê-lo você estará colaborando para que possamos melhorar os cursos da sua área, através de novas atividades de ensino, que serão elaboradas a partir dele. 
Não há necessidade de identificação do seu nome. MUITO OBRIGADO!

Favor assinalar com "x" a alternativa que se aplica ao seu caso:

\subsection{Sexo $\square$ Feminino $\square$ Masculino}

1.2 Idade:

1.3 Ensino Médio frequientado: $\square$ Todo em escola particular $\square$ Todo em escola pública $\square$ em escolas públicas e privadas.

1.4 Recebe bolsa: $\square$ escola da família $\square$ da instituição $\square$ outras.

1.5 Trabalha: $\square \operatorname{sim} \square$ não

1.6 Grau de instrução do pai: $\square$ Até $8^{\mathrm{a}}$ série $\square$ Ensino Médio $\square$ Ensino superior $\square$ Pósgraduação.

1.7 Grau de instrução da mãe: $\square$ Até $8^{\mathrm{a}}$ série $\square$ Ensino Médio $\square$ Ensino superior $\square$ Pósgraduação.

Nas questões abaixo, assinale com um $\mathrm{X}$ a lacuna, que mais está em concordância com o que você pensa ou acredita. As lacunas correspondem a: CP: Concordo Plenamente; C: Concordo; I: Indiferente; D: Discordo DP: Discordo Plenamente.

\begin{tabular}{|c|c|c|c|c|c|}
\hline ESCOLHI O CURSO DE PEDAGOGIA PORQUÊ: & $\mathbf{C P}$ & $\mathbf{C}$ & I & $\mathbf{D}$ & DP \\
\hline \multicolumn{6}{|l|}{ 01) procuro aperfeiçoamento profissional. } \\
\hline \multicolumn{6}{|l|}{ 02) procuro progresso profissional. } \\
\hline \multicolumn{6}{|l|}{ 03) preciso melhorar minha qualificação profissional. } \\
\hline \multicolumn{6}{|l|}{ 04) preciso aprimorar meu currículo. } \\
\hline \multicolumn{6}{|l|}{ 05) em função das exigências da nova LDB 9394/96. } \\
\hline \multicolumn{6}{|l|}{ 06) quero abrir uma escola. } \\
\hline \multicolumn{6}{|l|}{ 07) quero legalizar minha escola. } \\
\hline \multicolumn{6}{|l|}{ 08) tenho muito interesse pela área. } \\
\hline \multicolumn{6}{|l|}{ 09) tenho vocação para ser Pedagogo (a). } \\
\hline \multicolumn{6}{|l|}{ 10) quero ser professor (a). } \\
\hline \multicolumn{6}{|l|}{ 11) o curso é voltado para a área das humanidades. } \\
\hline \multicolumn{6}{|l|}{ 12) não fiz magistério. } \\
\hline \multicolumn{6}{|l|}{ 13) para ter um curso de nível superior. } \\
\hline \multicolumn{6}{|l|}{ 14) para poder trabalhar na área de treinamento de pessoal. } \\
\hline \multicolumn{6}{|l|}{ 15) quero me especializar para trabalhar com crianças. } \\
\hline \multicolumn{6}{|l|}{ 16) o curso oferece muitas opções de trabalho. } \\
\hline \multicolumn{6}{|l|}{ 17) para trabalhar em treinamento empresarial } \\
\hline \multicolumn{6}{|l|}{ 18) quero ser diretor (a). } \\
\hline \multicolumn{6}{|l|}{ 19) quero ser orientador (a) educacional. } \\
\hline \multicolumn{6}{|l|}{ 20) quero ser supervisor (a) de ensino. } \\
\hline \multicolumn{6}{|l|}{ 21) quero ter mais informações sobre a área. } \\
\hline \multicolumn{6}{|l|}{ 22) quero melhorar minha prática educativa. } \\
\hline 23) quero melhorar o ensino nas escolas públicas. & & & & & \\
\hline
\end{tabular}




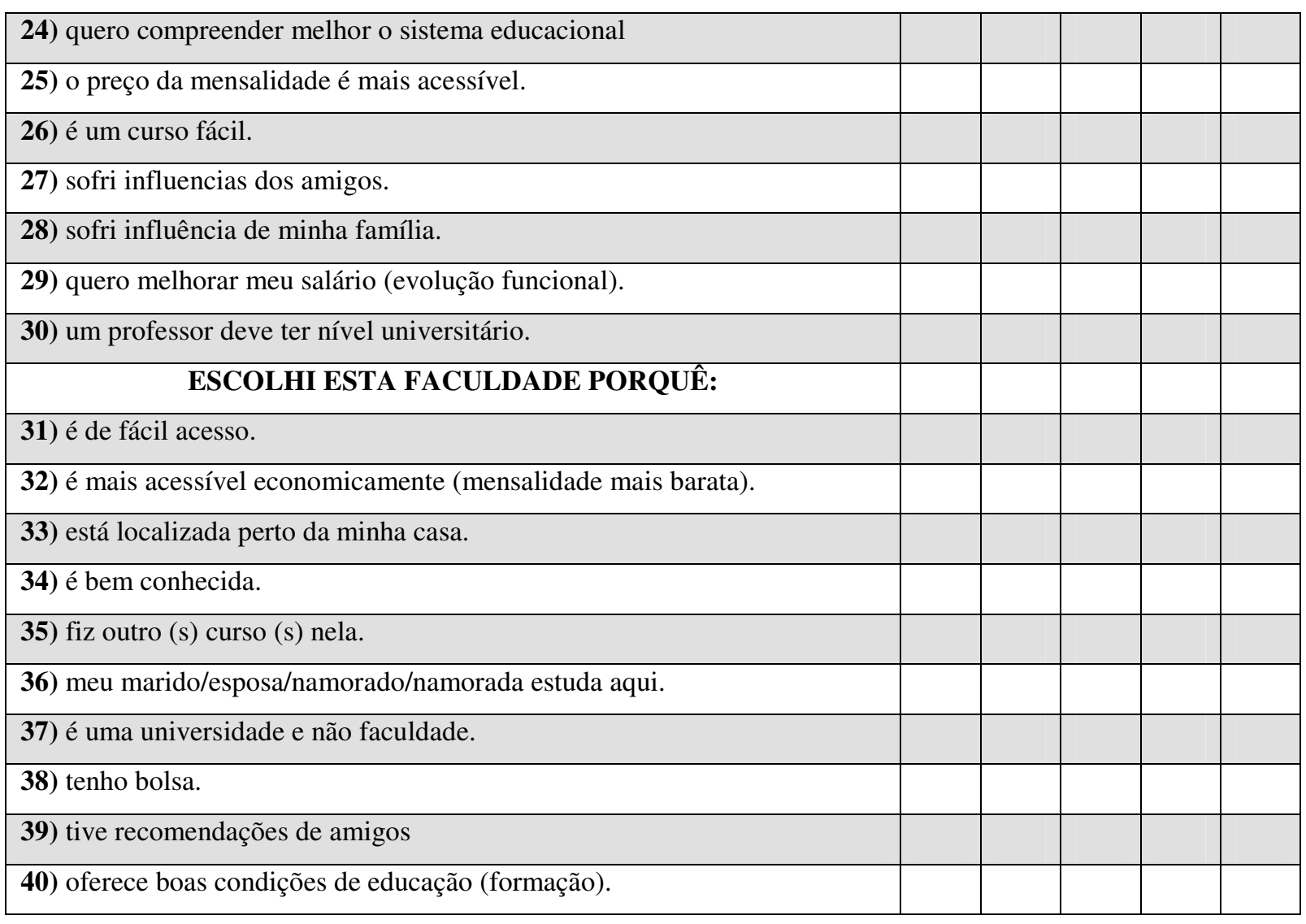

